6-1-2013

\title{
Operative management of partial-thickness tears of the proximal hamstring muscles in athletes.
}

\author{
Karl F Bowman \\ Harbour Sports Medicine, Wardell Orthopaedics \\ Steven B. Cohen \\ Thomas Jefferson University \\ James P Bradley \\ Burke and Bradley Orthopaedics, UPMC Center for Sports Medicine
}

Follow this and additional works at: https://jdc.jefferson.edu/rothman_institute Let us know how access to this document benefits you

\section{Recommended Citation}

Bowman, Karl F; Cohen, Steven B.; and Bradley, James P, "Operative management of partialthickness tears of the proximal hamstring muscles in athletes." (2013). Rothman Institute Faculty Papers. Paper 53.

https://jdc.jefferson.edu/rothman_institute/53

This Article is brought to you for free and open access by the Jefferson Digital Commons. The Jefferson Digital Commons is a service of Thomas Jefferson University's Center for Teaching and Learning (CTL). The Commons is a showcase for Jefferson books and journals, peer-reviewed scholarly publications, unique historical collections from the University archives, and teaching tools. The Jefferson Digital Commons allows researchers and interested readers anywhere in the world to learn about and keep up to date with Jefferson scholarship. This article has been accepted for inclusion in Rothman Institute Faculty Papers by an authorized administrator of the Jefferson Digital Commons. For more information, please contact: JeffersonDigitalCommons@jefferson.edu. 


\title{
OPERATIVE MANAGEMENT OF PARTIAL THICKNESS TEARS OF THE PROXIMAL HAMSTRINGS IN ATHLETES
}

\author{
Karl F Bowman Jr, MD \\ Fellow, Sports Medicine \\ UPMC Center for Sports Medicine \\ 3200 South Water Street \\ Pittsburgh, PA 15203 \\ Phone (734) 358-0169 \\ Fax (412) 432-3690 \\ Steven B Cohen, MD \\ Associate Professor \\ Rothman Institute \\ Thomas Jefferson University \\ 925 Chestnut Street \\ Philadelphia, PA 19107 \\ James P Bradley, MD (Corresponding author) \\ Clinical Professor \\ Burke and Bradley Orthopaedics \\ UPMC Center for Sports Medicine \\ 200 Delafield Road, Suite 2010 \\ Pittsburgh, PA 15215 \\ Phone (412) 784-5770 \\ Fax (412) 784-5776
}




\begin{abstract}
Background: Chronic insertional tendinopathy of the hamstring origin represents a challenging clinical problem to the orthopaedist, and can lead to significant impairment of recreational and competitive athletic performance. Non-operative treatment is frequently met with limited success, while there is a paucity of data on the efficacy of surgical management for partial proximal hamstring tears in the setting of tendinopathy.
\end{abstract}

Purpose: To evaluate the results of operative treatment for partial tears of the hamstring origin in athletes in the setting of underlying tendinopathy.

Study Design: Case series; Level of Evidence, 4.

Methods The records of 17 patients with chronic hamstring tendinopathy and partial tearing were reviewed after IRB approval was obtained. All patients were treated with open debridement and primary tendon repair after failure of an appropriate course of nonoperative therapy. Clinical and operative records, radiographs and MR imaging were reviewed for all patients. A patient reported outcomes survey was completed by 14 patients including the Lower extremity functional score (LEFS), Marx activity rating scale, and subjective patient satisfaction scores. Early and late post-operative complications were recorded.

Results: Patients included 3 males and 14 females with an average age of 43 years 4 months (range, 19-64y) and an average follow-up of 32 months (12-51m). Post-operative LEFS was $73.3 \pm 9.9$ (56-80), average Marx activity scores was $6.5 \pm 5.3$ (0-16). No patient underwent a subsequent surgery on their hamstring, although 1 patient was not satisfied with their surgical result. Complications include 1 superficial suture abscess treated with dressing changes and 1 patient with residual foot numbness without motor deficit. Two patients had a recurrence of symptoms following return to activities treated with physical therapy and local injection. All patients were able to return to their preoperative level of activity, although a single patient reported persistent symptoms during competitive distance running.

Conclusions: Surgical treatment of partial proximal hamstring tears in the setting of tendinopathy can lead to satisfactory functional outcomes, a high rate of return to athletic activity, and low complication rate. This procedure should be reserved for patients who have failed an extended course of non-operative treatment, and the proximity of the sciatic nerve mandates a careful assessment of the risk/benefit ratio before proceeding to the operating room.

Keywords: Hamstring avulsion, tendinopathy, hamstring syndrome, ischial tuberosity, sciatic nerve 


\section{Introduction}

Injuries to the proximal hamstring musculature are frequently encountered in the athletic population. These injuries occur on a spectrum of severity and can range from a minor sprain to a complete proximal avulsion necessitating surgical repair. The most common mechanism is eccentric lengthening of the muscle unit during the terminal swing phase of gait during explosive activities involving rapid limb acceleration and deceleration such as sprinting or cutting maneuvers (reference on swing and injury). The hamstring muscle group is particularly susceptible to injury as three of the four major components of the complex cross both the hip and knee joint. Injury most commonly involves the tendinous origin at the ischial tuberosity, although these injuries may also occur intramuscularly or at the musculotendinous junction [1]. The origin of the hamstring muscle group includes the posterior and medial attachment of the common tendon of the long head of the biceps femoris and semitendinosus muscle at an oval footprint measuring approximately $2.7 \mathrm{~cm}$ $\mathrm{x} 1.8 \mathrm{~cm}$, and the crescent shaped attachment of the semimembranosus muscle at the inferior and lateral aspect of the ischial tuberosity measuring $3.1 \mathrm{~cm} \times 1.1 \mathrm{~cm} \mathrm{[2].} \mathrm{The}$ short head of the biceps femoris inserts on the proximal femur at the linea aspera. Injury to the hamstring origin most commonly involves the biceps femoris and semitendinosus, with the semimembranosus least commonly involved $[1,3,4]$.

Patients can also develop symptomatic pain at the hamstring origin without acute injury or tearing. This is generally considered to be part of the spectrum of overuse injuries and has been previously labeled as "Hamstring Syndrome"(reference). In contrast to acute injuries, tendinopathy occurs more commonly at the origin of the semimembranosis with adjacent tendon involvement occurring less frequently (reference). Treatment options initially consist of non-operative modalities for management of symptoms including activity modification, non-steroidal anti-inflammatory medication, and shockwave therapy, and local injection with either corticosteroid or platelet rich plasma. Despite these various options the management of symptomatic proximal hamstring tendinopathy presents a clinical challenge to the orthopaedist and often results in a prolonged and frustrating treatment course for the patient $[5,6]$. Lempainen and colleagues published their results on surgical management for recalcitrant cases and reported an $89 \%$ good to excellent result allowing return to sport at the pre-injury level. Their surgical procedure involved resecting the proximal 3 to 4 centimeters of the semimembranosus and performing a side-to-side tenodesis to the neighboring biceps femoris.

Recent literature has focused on the identification and treatment of these injuries with many authors now recommending acute repair for complete proximal hamstring ruptures in athletes[7]. Surgical treatment of these patients has demonstrated higher patient satisfaction, improved recovery of hamstring strength and higher return to previous activity levels $[8,9]$. There has been an increasing awareness of partial avulsions of the proximal origin without retraction and its progression to activity related pain and limb dysfunction that can limit return to competitive athletics. There remains a paucity of studies characterizing the outcomes of surgical treatment of partial proximal hamstring avulsions [3]. The purpose of this study is to describe the outcomes following operative management of partial avulsion of the proximal origin of the hamstring musculature in 
athletes. 


\section{Materials and Methods}

\section{-inclusion criteria}

This is a retrospective review of all patients who underwent operative management of painful proximal hamstring tendinopathy that were found on MRI to have partial tearing without retraction by the senior author (JPB) between 2007 and 2010 with at least one year of follow-up. Institutional Review Board approval was obtained prior to collection of patient data. Exclusion criteria included any patient with a complete tear of the hamstring origin involving all three tendons (biceps femoris, semimembranosus and semitendinosus). Patient demographics were obtained from the medical record, radiographic and magnetic resonance imaging studies and operative reports. All patients included in our study failed at least six months of nonoperative management including activity modification, non-steriodal anti-inflammatory treatment if medically indicated, formal physical therapy, and image guided injection. Failure of non-operative treatment was defined as persistent symptoms of proximal thigh pain or spasm/cramping at rest or inability to return to previous level of athletic activity due to symptoms in the presence of chronic hamstring tendinopathy and partial thickness tearing without retraction of the hamstring origin at the ischial tuberosity identified on MRI. The timing of surgery was based on patient's preference, activity level and restrictions due to symptoms, and pain level.

\section{-Physical Exam}

Patients with a clinically suspected acute or chronic injury to the proximal hamstring were examined for evidence of posterior thigh ecchymosis, palpable muscular gapping, and focal tenderness at the ischial tuberosity on the affected side. Objective strength testing is performed and compared to the contralateral side with resisted knee flexion at 90, 45 and 20 degrees of knee flexion, active single limb pelvic elevation with the affected leg, and standing heel drag testing. Objective findings of weakness and subjective complaints of pain are recorded. Hamstring flexibility, popliteal angle, and a detailed neurological examination of the affected extremity were also performed and recorded.

\section{-Imaging-}

At the time of initial presentation, all patients initially undergo radiographic imaging including an AP and frog-lateral pelvis radiograph to assess for ischial apophyseal avulsion, ischial enthesopathy, or fracture. In clinically suspected cases of proximal hamstring pathology, MR imaging is obtained utilizing a torso coil with bilateral imaging from the mid-thigh to above the ischial tuberosities. Sequences include Coronal T1 and STIR, axial T1 without fat suppression, axial T2 with and without fat suppression, and T1 sagittal and T2 sagittal images. A vitamin E capsule is placed over the site of discomfort for localization of pathology. Partial tears of the proximal hamstring origin were identified by a fellowship trained musculoskeletal radiologist and discussed with the treating surgeon prior to discussion of treatment options. Partial thickness tearing of the 
proximal hamstring complex was identified by a crescent shaped lesion within the tendinous origin of the proximal hamstring best seen on coronal T2-weighted images. We identified this finding as the "sickle-sign" and found it to be pathognomonic for partial avulsion of the proximal hamstring origin (Figure XX).

\section{-Surgical technique}

Following the induction of general anesthesia and administration of appropriate preoperative prophylactic antibiotics, the patient is placed prone on a standard operative table. All bony prominences are well padded with the hips placed over the center of the bed. The bed is then flexed to allow 20 degrees of hip flexion in order to facilitate the surgical exposure. The perineum is sealed off with an adhesive impervious barrier and the entire extremity is surgically prepped and draped. Loupe magnification and a surgical headlight are utilized to improve visualization and identification of local neurovascular structures. A transverse incision is placed along the gluteal crease, centered over the ischial tuberosity. Superficial dissection is performed sharply with care taken to protect the sensory branches of the posterior femoral cutaneous nerve. The gluteal fascia is then incised longitudinally in line with the skin incision. We have recently begun performing a gluteus maximus splitting approach to facilitate exposure, especially in muscular patients. There are several intramuscular fascial layers that must be identified and incised during this muscle splitting technique. Care must also be taken to identify and protect any branches of the inferior gluteal nerve that may be encountered during the exposure. The sciatic nerve is palpated and protected during the approach, but rarely is a neurolysis required unless there are pre-operative symptoms of sciatic irritation or evidence of significant fibrosis involving the nerve. The ischial tuberosity is then located and exposed with curved Dever retractors placed medially, laterally, superiorly and inferiorly. We avoid the use of sharp or self-retaining retractors to prevent iatrogenic injury to the sciatic nerve. In the case of partial tendinous avulsions of the proximal hamstring, there will often be normal appearing tissue overlying the tuberosity that must be elevated to reveal the underlying partial injury. This has been previously described as the "hidden lesion" (Sallay). Chronic-appearing or attenuated tissue associated with a longstanding partial tear must be debrided and the tendon edges freshened to healthy tendon. The ischial tuberosity footprint is also identified and cleared of scarred or attenuated tissue, followed by gentle decortication with a rasp, curette, or scored with an osteotome. We avoid the use of a motorized burr to minimize the risk of iatrogenic neurovascular injury. The number and configuration of suture anchors depends on the amount of the hamstring origin that is not avulsed. If the entire footprint is involved we will elevated and debride the entire insertion followed by placement of 5 suture anchors $(2.8 \mathrm{~mm}$ Arthrex Bioroc Suture Anchors, Naples FL) in an "X" pattern as previously described [7]. Although rare, if a significant portion of the native footprint is intact we would only repair the involved tissue. The knee is flexed to 30 degrees and the sutures are passed in a retrograde fashion with a needle driver or a commercially available suture passing device (Arthrex Fastpass Scorpion Suture Passer, Naples FL) and tied in a horizontal mattress configuration from proximal to distal to allow as much proximal advancement of the tissue as possible while reapproximating tissue to the entire hamstring origin footprint. A side to side repair of the avulsed tendon to the intact hamstring is performed if necessary. 
The wound is irrigated and hemostasis is obtained. The wound is closed in a layered fashion with particular attention placed on achieving a water-tight closure of the gluteal fascia to avoid the development of a post-operative hematoma or seroma. The affected leg is then placed into a knee immobilizer in 20-40 degrees of locked flexion for a period of 6 weeks to take tension off of the hamstring and protect the surgical repair. The patient is allowed to perform toe touch weight bearing with crutch assistance and aspirin is utilized for DVT prophylaxis for 4 weeks following surgery. Passive hip range of motion is initiated with a therapist at 2 weeks and active hip flexion is allowed at 4 weeks in conjunction with core strengthening. Isotonic strengthening is started at 6 weeks and isokinetic strengthening is added at 8 week post-operatively. The brace is removed at 6-8 weeks post-operatively. Dry land training and gentle sport-specific activity is initiated at 12 weeks with return to unrestricted activity allowed no earlier than 6 months following surgery.

\section{-Questionnaire}

Patients who met the inclusion criteria were contacted via telephone, office examination, or email correspondence. A standardized survey was completed by each patient that was able to be contacted. Outcome measures included the Marx knee activity rating scale[10], the Lower Extremity Functional Scale (LEFS) [11]. Additional questions included in the survey evaluated mechanism of injury, subjective lower extremity strength compared to the contralateral limb, the presence of posterior thigh or foot numbness during activity, time from symptom onset until medical evaluation, time from initial evaluation until surgical recommendation, and overall patient satisfaction with the post-operative results. The complete questionnaire can be found in the appendix.

\section{-Statistics}

Patient data was collected in a spreadsheet database and statistical calculations were performed within the spreadsheet program (Microsoft Excel, Redmond WA). 


\section{Results}

-demographics (age, activity level, follow-up)

A review of 17 cases of operative treatment for chronic proximal hamstring tendinopathy with associated partial thickness tendon tearing was performed. Surgeries were performed by the senior author (JPB) over a 3 year period (2007-2010). 14 patients were able to be contacted and agreed to complete a survey regarding current functional outcomes on the operative leg, overall satisfaction with the surgical intervention, and subjective grading of operative limb strength compared to the contralateral side. Additional subjective comments were also recorded. There were no patients who underwent bilateral operative treatment. Three patients were not able to be contacted after multiple attempts and information reported in this study was obtained entirely from a review of their medical records. Patients included 3 males and 14 females with an average age of 43 years 4 months (range, 19-64 years) and an average of 32 months follow-up (12-51 months). There were 8 procedures performed on the left leg and 9 performed on the right leg. No patients underwent bilateral surgical intervention at the time of publication. Athletic participation included two collegiate athletes (field hockey, track and field), and 14 recreational athletes (waterskiing, distance running, tennis) and a professional bodybuilder. Fourteen patients reported an acute exacerbation of chronic symptoms sustained while engaging in exercise or sport specific activity. The most common mechanism of injury was an eccentric injury during hip flexion and knee extension. Fifteen patients were initially managed by another medical provider prior to referral to our office for recalcitrant symptoms. All patients underwent a minimum of 6 months of nonoperative treatment for including physical therapy, activity modification, and selective injection with either corticosteroid or autologous conditioned plasma (ACP, Arthrex- Naples, FL).

Clinical examination was positive for focal tenderness to palpation at the ischial tuberosity and proximal hamstring origin in all patients without evidence of palpable defect. Symptoms were routinely exacerbated with hip flexion while the knee was maintained in an extended position. Strength testing revelaed no worse than $4 / 5$ strength when compared to the contralateral leg. All patients were able to perform standard activities of daily living (ADL's), but complained of localized proximal and posterior thigh pain with activity and prolonged periods of sitting or lying on the affected side.

-survey results

The results of the Marx Activity Scale post-operatively averaged $6.5 \pm 5.3$ out of a maximal score of 16 (range 0-16), and the Lower Extremity Functional Score averaged $73.3 \pm 9.8$ out of a maximum score of 80 (range 56-80). The Marx activity Style score was 20 out of a maximal score off 20 for all patients. The LEFS style score average 66.7 \pm 17.0 out of 80 (range $36-80$ ).

-subjective results 
The subjective patient responses generated in the survey identified that 13 of 14 patients were satisfied with their post-operative results at the time of latest follow-up. Subjective strength was estimated to be an average of $89 \%$ (range $75-100 \%$ ) when compared to the contralateral leg. Ten patients reported the ability to return to strenuous activities including sports without limitation from their hamstring surgery, 1 patient reported intermittent limitations with strenuous activity and 3 patients reported that they were not able to participate in strenuous activities as a result of their hamstring surgery. One patient reported that their operative leg continued to affect their activity level daily, two patients reported interference with activities one to three times monthly, five patients reported symptoms less than one time per month, and six patients reported no limitations with daily activities. One patient experienced cramping in the affected extremity weekly, one patient reported cramping up to 3 times per month, 4 patients experienced cramping less than once per month, and eight patients reported no episodes of cramping postoperatively. No patients reported difficulty with loss of leg control during brisk jogging or running.

The proximity of the posterior femoral cutaneous and sciatic nerves to the proximal hamstring place these structures at risk during operative intervention. Post-operatively, two patients reported posterior thigh parathesias at rest, one patient experienced parathesias during daily activities and one patient experienced these symptoms only during strenuous exercise. Two additional patients reported parathesias including numbness and burning in the ipsilateral foot at rest and with strenuous exercise. The cutaneous surgical scar and prominence of the tendinous repair have also been identified as sources of discomfort post-operatively. Seven patients reported no restrictions with prolonged sitting on the operative extremity, one patient reported not being able to sit comfortably beyond 4 hours, three patients were uncomfortable after 1 hour, and 3 patients experienced discomfort after 30 minutes.

\section{-Failures}

Two patients had a recurrence of their symptoms after returning to their pre-operative activity level requiring additional intervention. One patient was a competitive distance runner with 36 month follow-up who had experienced several years of symptoms associated with competition prior to surgical intervention. Post-operatively, this patient reported daily persistent painful symptoms although they were able to return to competition. There was no associated reported cramping, loss of leg control, or distal numbness/tingling although they were not able to sit comfortably for longer than 30 minutes. This patient elected to undergo image guided autologous conditioned plasma (ACP- Arthrex, Naples FL), which did not significantly improve their symptoms. The second patient reported a minor recurrence of symptoms bilaterally four months postoperatively during the initiation of strengthening and sport specific activities. Bilateral image guided ACP injections were performed at that time with resolution of symptoms and subsequent return to recreational softball. Overall, only one patient reported not being satisfied with their post-operative results at final follow-up. 
Immediate post-operative complications include a single wound complication consisting of a small stitch abscess. This was treated with local debridement in clinic and resulted in uneventful wound healing with twice daily dressing changes. A patient developed numbness in an L4 distribution without focal motor weakness at 6 months following surgery during rehabilitation centered on sport specific training. Subsequent lumbar and proximal hamstring imaging identified an intervertebral disc herniation at the L3 level. This patient had successful resolution of symptoms with a modification of therapy exercises and a single epidural steroid injection. No subsequent surgeries on the ipsilateral or contralateral hamstring were performed. 


\section{Discussion}

Chronic insertional tendinopathies of the lower extremity represent a challenging clinical problem to the orthopaedist. The causes and pathophysiology of tendinopathies has been extensively studied but not scienfitically proven. It is commonly considered to be a result of a failed or incomplete healing response to repetitive tearing or stretching associated with overuse activities. The hamstring musculature is particularly prone to the development of tendinopathy associated with overuse as it crosses both the knee and hip joints and undergoes repetitive concentric and eccentric contractions with athletic activities such as running and provides explosive force during rapid acceleration and deceleration encountered in sprinting and cutting activities[12]. Tendinopathy of the hamstrings is frequently associated with individuals who maintain an active life style and can be very frustrating for both patient and physician, as it can lead to significant impairment of recreational and competitive athletic performance. Initial treatment strategies should always include an appropaite course of non-operative management prior to consideration of surgical intervention. These treatment options include initial activity modification and avoidance of aggravating factors. Selective use or oral antiinflammatories can also assist in providing relief from symptoms. Initiation of a lower extremity and back stretching program with soft tissue mobilization can then be added followed by eccentric hamstring strengthening and core strengthening prior to initiation of activity specific exercises and return to athletics. If symptoms persist or recur, it is not uncommon to utilize image guided local injection with corticosteroid Limited success has been achieved with various non-operative treatment modalities including activity modification and local injection with corticosteroid or platelet rich plasma [5]. Despite these various options the management of symptomatic proximal hamstring tendinopathy presents a clinical challenge to the orthopaedist and often results in a prolonged and frustrating treatment course for the patient. Previous studies have reported the results of surgical management for complete traumatic tendinous avulsions of the proximal hamstring musculature [7, 13-15], but a paucity of literature is available on the results of surgical repair of partial thickness hamstring tears with underlying chronic tendinopathy $[3,16,17]$. The results of our study does provide further information on the expected post operative outcomes following surgical treatment of chronic proximal hamstring tendinopathy with partial thickness tearing an a healthy athletic population.

Puranan surgical procedure

Lempainen and colleagues published their results on surgical management for recalcitrant cases and reported an $89 \%$ good to excellent result and were able to return to sport at the same level. The surgical procedure involved resecting the proximal 3 to 4 centimeters of the semimembranosus and performing a side-to-side tenodesis to the neighboring biceps femoris. This intervention could potentially fail to address the pathology of the biceps femoris and semitendinosis insertions and may predispose to symptomatic recurrence. A side-to-side repair may also decrease the insertional strength of the hamstring origin at the ischial tuberosity and lead to future complete rupture. Our technique involves taking down the entire hamstring insertion and debriding all involved inflammatory tissue and 
performing an anatomic repair of all three tendon components in a manner that allows complete restoration of the native insertion without tissue prominence or bunching.

There are several limitations to this study, with the most obvious being the retrospective nature and limited number of patients. Chronic proximal hamstring tendinopathy is a relatively uncommon complaint and that is primarily treated with non-operative modalities with intermittent success. Patients will also frequently modify their activities to the point of avoiding aggravating factors and discontinuing athletic activities. Surgical intervention is reserved for prolonged or recalcitrant symptoms in patients who are not able to modify their activity levels or report daily symptoms. We have further limited our surgical indications to cases with MRI evidence of partial thickness tearing of the hamstring origin without retraction in the setting of chronic tendinopathy. The majority of our patients received multiple previous opinions prior to surgical consultation, which makes it difficult to accurately select a non-operative comparison cohort for a prospective or comparative retrospective study.

-Use of Marx activity score 


\section{References}

1. De Smet, A.A. and T.M. Best, MR imaging of the distribution and location of acute hamstring injuries in athletes. AJR Am J Roentgenol, 2000. 174(2): p. 3939.

2. Miller, S.L., J. Gill, and G.R. Webb, The proximal origin of the hamstrings and surrounding anatomy encountered during repair. A cadaveric study. J Bone Joint Surg Am, 2007. 89(1): p. 44-8.

3. Lempainen, L., et al., Surgical treatment of partial tears of the proximal origin of the hamstring muscles. Br J Sports Med, 2006. 40(8): p. 688-91.

4. Koulouris, G. and D. Connell, Evaluation of the hamstring muscle complex following acute injury. Skeletal Radiol, 2003. 32(10): p. 582-9.

5. Zissen, M.H., et al., High hamstring tendinopathy: MRI and ultrasound imaging and therapeutic efficacy of percutaneous corticosteroid injection. AJR Am J Roentgenol. 195(4): p. 993-8.

6. Cacchio, A., et al., Shockwave therapy for the treatment of chronic proximal hamstring tendinopathy in professional athletes. Am J Sports Med. 39(1): p. 14653.

7. Cohen, S. and J. Bradley, Acute proximal hamstring rupture. J Am Acad Orthop Surg, 2007. 15(6): p. 350-5.

8. Harris, J.D., et al., Treatment of proximal hamstring ruptures - a systematic review. Int J Sports Med. 32(7): p. 490-5.

9. McGregor, C., et al., Traumatic and overuse injuries of the ischial origin of the hamstrings. Disabil Rehabil, 2008. 30(20-22): p. 1597-601.

10. Marx, R.G., et al., Development and evaluation of an activity rating scale for disorders of the knee. Am J Sports Med, 2001. 29(2): p. 213-8.

11. Binkley, J.M., et al., The Lower Extremity Functional Scale (LEFS): scale development, measurement properties, and clinical application. North American Orthopaedic Rehabilitation Research Network. Phys Ther, 1999. 79(4): p. 371-83.

12. Fredericson, M., et al., High hamstring tendinopathy in runners: meeting the challenges of diagnosis, treatment, and rehabilitation. Phys Sportsmed, 2005. 33(5): p. 32-43.

13. Brucker, P.U. and A.B. Imhoff, Functional assessment after acute and chronic complete ruptures of the proximal hamstring tendons. Knee Surg Sports Traumatol Arthrosc, 2005. 13(5): p. 411-8.

14. Sarimo, J., et al., Complete proximal hamstring avulsions: a series of 41 patients with operative treatment. Am J Sports Med, 2008. 36(6): p. 1110-5.

15. Wood, D.G., et al., Avulsion of the proximal hamstring origin. J Bone Joint Surg Am, 2008. 90(11): p. 2365-74.

16. Lempainen, L., et al., Proximal hamstring tendinopathy: results of surgical management and histopathologic findings. Am J Sports Med, 2009. 37(4): p. 72734.

17. Puranen, J. and S. Orava, The hamstring syndrome. A new diagnosis of gluteal sciatic pain. Am J Sports Med, 1988. 16(5): p. 517-21. 
